\title{
28 Möglichkeiten der Dissemination in einem Verbundprojekt
}

\author{
Daniel Köhler
}

Die im optes-Verbundprojekt entwickelten propädeutischen Angebote wurden in erster Linie für die zum Verbundprojekt zugehörigen Hochschulen mit dem Ziel entwickelt, die Studienabbruchquoten bei Studienanfänger*innen insbesondere bei MINT-Studiengängen durch die Nacharbeitung fehlender Mathematikkenntnisse zu senken.

Je nach Studiengang ergaben sich hier bei den Verbundpartnern aber unterschiedliche Anforderungen, Schwerpunkte und Gestaltungswünsche an das jeweilige einzusetzende lokale mathematische Propädeutikum (mathematische Brückenkurse zur Studienvorbereitung).

Dennoch bestand von Anfang an die Idee, die entwickelten optes-Angebote untereinander auszutauschen (interne Dissemination), gemeinsam weiterzuentwickeln, im Kern auf einen Standard zu heben und letztendlich der interessierten Öffentlichkeit zur Verfügung zu stellen (externe Dissemination).

Die Entwicklung der jeweiligen optes-Angebote wurde dezentral auf die Verbundpartner verteilt, sodass jedes Angebot, zum Beispiel die mathematischen Selbstlernkurse, von einer eigenständigen „Fachabteilung“ (bei optes in Form von Teilprojekten) erstellt wurde. Diese Praxis der spezifischen Angebotserstellung durch jeweils zuständige Teilprojekte hatte sich bewährt, da hier Kompetenzen konzentriert und gebündelt wurden.

Auf Quartalstreffen und Videokonferenzen wurde dann gemeinsam im Gesamtverbund die Richtung festgelegt, sodass die entsprechenden optes-Angebote unter einem gemeinsamen Konsens entwickelt werden konnten.

Die daraus resultierenden und unter einem Qualitätsstandard entstandenen optes-Angebote wurden dann von den pilotierenden Hochschulen für ihre lokalen Propädeutika hin individualisiert. Diese Individualisierungen betrafen systemseitige Einstellungen in ILIAS sowie teilweise auch Anpassungen an das Corporate Design der jeweiligen Hochschule. Jede Hochschule entschied dabei selbst, welches optes-Angebot in welchem Umfang eingesetzt wurde.

Die Originalversion dieses Kapitels wurde revidiert. Ein Erratum ist verfügbar unter https://doi.org/10.1007/978-3-658-31279-4_32 
Bevor ein optes-Angebot an die Verbundpartner intern disseminiert wurde, durchlief es eine umfangreiche Pilotierung und Evaluierung vom erstellenden Teilprojekt. Dieser Prozess wurde eng begleitet von den Teilprojekten „Prozessbegleitung und didaktische Beratung“, sowie vom Teilprojekt „Qualitätssicherung“.

Somit wurde im optes-Verbundprojekt ein Qualitätsstandard für neu entwickelte Angebote sichergestellt, der auch für die externe Dissemination einen immensen Mehrwert bot: Es wurde nichts veröffentlicht, dass nicht zuvor getestet und verbessert wurde. Die aus den Evaluationen gewonnenen Erkenntnisse flossen dann in zahlreiche Verbesserungsmaßnahmen der jeweiligen optes-Angebote mit ein. Diese Praxis bewährte sich sowohl für die interne als auch die externe Dissemination.

Jedoch ergaben sich sowohl für die interne als auch die externe Dissemination oftmals Schwierigkeiten in der Einhaltung von Zeitplänen. Die Entwicklungs-, Pilotierungs- und Überarbeitungsphasen waren relativ lang, um die optes-Angebote mit den verfügbaren Ressourcen in der gewünschten Qualität zu erstellen. Zusätzlich gab es durch Abhängigkeiten von Zuarbeiten anderer Teilprojekte mitunter Verzögerungen, die im ursprünglichen Projektplan nicht vorgesehen waren. Das Teilprojekt Dissemination konnte damit die optes-Angebote für die externe Dissemination erst relativ spät in der Projektphase übernehmen.

Im optes-Verbundprojekt war das Teilprojekt Dissemination zuständig für die externe Dissemination an die interessierte Öffentlichkeit. In diesem Teilprojekt wurden die Projektergebnisse der einzelnen Teilprojekte gebündelt, für die Öffentlichkeit aufbereitet und an diese veröffentlicht. Vereinfacht gesagt, übernahm das Teilprojekt Dissemination im optes-Gesamtprojekt die Rolle des Vertriebs- und Marketings sowie sonstige öffentlichkeitswirksame Aufgaben. Da die intern entwickelten optes-Angebote an die Anforderungen der teilnehmenden Hochschulen angepasst wurden, befasste sich das Teilprojekt Dissemination mit der Entwicklung eines Qualitätsstandards für die Öffentlichkeit und stimmte diesen mit den Teilprojekten $a b$.

$\mathrm{Zu}$ diesem Qualitätsstandard gehörte ebenfalls, dass die Teilprojekte ihre optes-Angebote mit Standard-ILIAS-Features erstellen, sodass alle externen Anwender*innen die erstellten Inhalte auf ihren lokalen ILIAS-Installationen auch nutzen können. 
Grundsätzlich stand die Dissemination in einem engen Austausch mit den projektexternen, interessierten Hochschulen, sammelte Feedbacks und leitete diese wiederum ins optes-Projekt weiter.

Die Einholung von Feedbacks externer Anwender*innen stellte sich ebenfalls als sehr wertvolle Maßnahme heraus, da hier die optes-Angebote aus einer anderen Perspektive heraus bewertet wurden.

Die Dissemination an die interessierte Öffentlichkeit wird im nächsten Kapitel 29 näher betrachtet.

Open Access Dieses Kapitel wird unter der Creative Commons Namensnennung 4.0 International Lizenz (http://creativecommons.org/licenses/by/4.0/deed. de) veröffentlicht, welche die Nutzung, Vervielfältigung, Bearbeitung, Verbreitung und Wiedergabe in jeglichem Medium und Format erlaubt, sofern Sie den/ die ursprünglichen Autor(en) und die Quelle ordnungsgemäß nennen, einen Link zur Creative Commons Lizenz beifügen und angeben, ob Änderungen vorgenommen wurden.

Die in diesem Kapitel enthaltenen Bilder und sonstiges Drittmaterial unterliegen ebenfalls der genannten Creative Commons Lizenz, sofern sich aus der Abbildungslegende nichts anderes ergibt. Sofern das betreffende Material nicht unter der genannten Creative Commons Lizenz steht und die betreffende Handlung nicht nach gesetzlichen Vorschriften erlaubt ist, ist für die oben aufgeführten Weiterverwendungen des Materials die Einwilligung des jeweiligen Rechteinhabers einzuholen.

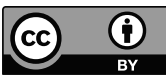

